\title{
UTILIZACIÓN DE LA POLIANILINA (PANI) EN LA SELECTIVIDAD DE IONES METÁLICOS POR MEDIO DE UN PROCESO DE ELECTRODIALISIS A TRAVES DE MEMBRANAS POLIMERICAS DE INTERCAMBIO IONICO
}

\section{USE OF POLYANILINE (PANI) IN THE SELECTIVITY OF METAL IONS BY MEANS OF A PROCESS OF ELECTRODIALYSIS THROUGH POLYMERIC MEMBRANES OF ION EXCHANGE}

\section{Doctorando:}

\section{César Jesús Espinoza}

Centro de Tecnologías Químicas, Facultad Regional Buenos Aires, Universidad Tecnológica Nacional - Argentina espinoza_cesar2@hotmail.com

\section{Director/es:}

\section{Juan Carlos Aphesteguy}

\section{Co-director/es:}

\section{María Cristina Di Stefano}

\section{Resumen}

El objetivo de este trabajo consiste en preparar mediante un proceso de síntesis electroquímica un polímero conductor: Polianilina (PANI). El mismo es caracterizado a través de espectroscopía infrarroja por transformada de Fourier (FTIR), por microscopía electrónica de barrido (SEM). Por último se efectúa un análisis de las ventajas comparativas de la síntesis electroquímica respecto de la oxidación química.

Palabras clave: polimero conductor, polianilina, polimerizacion electroquímica, ventajas

\begin{abstract}
The aim of this work is to prepare by means of a electrochemical synthesis process a conductive polymer: Polyaniline (PANI). It is characterized by Fourier transform infrared spectroscopy (FTIR), by scanning electron microscopy (SEM). Finally, an analysis is made of the comparative advantages of electrochemical synthesis with respect to chemical oxidation.
\end{abstract}

Keywords: conductor polymer, polyaniline, electrochemical polymerization, advantages

Introducción 
Los polímeros se comportan en general como aislantes eléctricos debido a la ausencia de electrones libres en su estructura capaces de participar en la conducción. Sin embargo se han logrado sintetizar materiales conductores de matriz polimérica llamados por ese motivo "metales sintéticos". Resultan ser tema de estudio debido a sus posibles aplicaciones en nanotecnología, biomedicina, sensores, membranas de intercambio, protección anticorrosiva, entre otros.

Pueden ser clasificados en polímeros conductores intrínsecos y extrínsecos. La conductividad en los intrínsecamente conductores (PCI) se origina en la conjugación extendida de electrones $\pi$ a lo largo de sus cadenas. Poseen átomos de carbono en la cadena principal con hibridación $\mathrm{sp}^{2}$. Esta determina enlaces $\sigma$ covalentes entre los carbonos de la cadena principal y los de las cadenas ramificadas dejando un orbital p no enlazado, solapándose para formar un enlace $\pi$, con una distribución de dobles enlaces $\mathrm{C}=\mathrm{C}$ alternados con los simples de C-C.

En este trabajo se considerarán únicamente los intrínsecamente conductores. Su comportamiento resulta más cercano al de los materiales semiconductores que al de los metales dado que sólo presentan altos valores de conductividad eléctrica bajo determinados estados de oxidación. Por cierta analogía con los semiconductores inorgánicos se hace referencia a los estados dopado y no dopado del polímero y a su vez, de procesos de dopado y desdopado. El dopado de un polímero implica la aparición de cargas eléctricas - electrones libres o huecos en su cadena principal, lo cual modifica las propiedades eléctricas propiciando la introducción de especies iónicas de carga opuesta llamadas contraiones. La diferencia entre los polímeros conductores y los semiconductores inorgánicos se basa en que para los primeros el dopado y desdopado se produce mediante reacciones rápidas y muchas veces reversibles entre moléculas discretas. Para los segundos en cambio las reacciones se producen en cristales inorgánicos en su estado sólido.

De acuerdo al uso se diferencian dos grupos fundamentales de materiales en base a las propiedades que utilizan para dar cumplimiento a sus funcionalidades:

Grupo I: Estas aplicaciones solo usan la conductividad de los polímeros facilitando el proceso de fabricación o simplemente el costo. Materiales electrostáticos, adhesivos conductores, blindaje electromagnético, placas de circuitos impresos, ropa antiestática, piezocerámica, electrónica activa (diodos, transistores).

Grupo II: este grupo utiliza la propiedad de electroactividad de los materiales. Electrónica molecular, pantallas eléctricas, sensores químicos, bioquímicos y térmicos, baterías recargables y electrolitos sólidos, sistemas de liberación de drogas, computadoras ópticas, membranas de intercambio iónico, actuadores electromecánicos, estructuras 'inteligentes', interruptores.

\section{Desarrollo}

\section{1-Espectroscopía infrarroja por transformada de Fourier}

Los productos obtenidos en la síntesis se estudiaron mediante la aplicación de la Espectroscopia Infrarroja por Transformada de Fourier (FTIR) para proceder a su caracterización e identificación.

Para llevar a cabo el estudio una pequeña porción de los productos de síntesis previamente desecados, se mezclan con bromuro de potasio ( $\mathrm{KBr}$ ) seco (invisible a la luz IR), en una proporción de 1:5. Para su correcta homogeneización se pasa la mezcla por mortero.

Las muestras así obtenidas se analizan en un Espectrómetro Infrarrojo por Transformada de Fourier (FTIR) Thermo Nicolet iS5. Se emplea un accesorio de Reflactancia Total Atenuada (ATR) de diamante con una región espectral ajustada en el rango de $4000-600 \mathrm{~cm}^{-1}$, número de scans 16 y resolución $4 \mathrm{~cm}^{-1}$.

Los resultados obtenidos se detallan a continuación: 


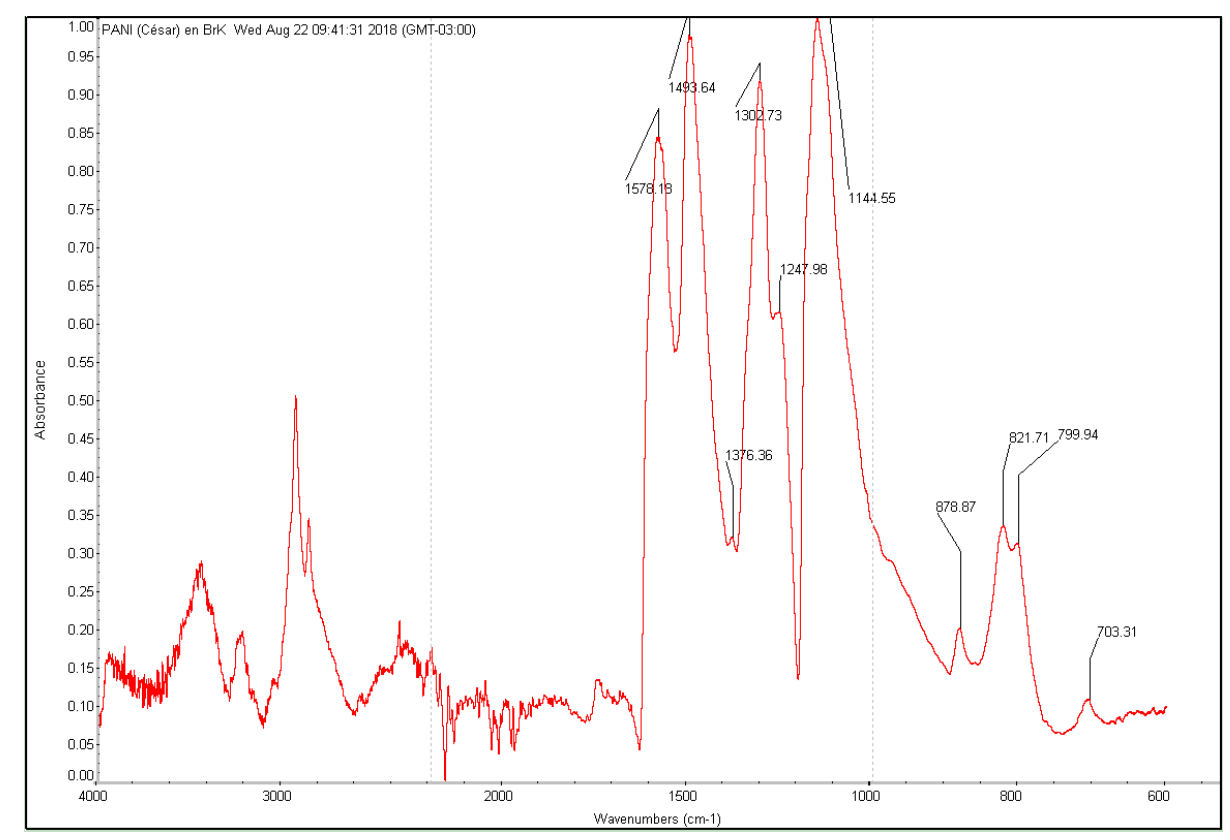

Figura 1: Espectro FTIR de polianilina (PANI) obtenida por método electroquímico empleando una corriente de 0,5 A

El espectro de referencia, de una muestra de polianilina (PANI) obtenida por método electroquímico (Figura 1) empleando una corriente de $0,5 \mathrm{~A}$, presenta dos bandas de absorción características, a $1578 \mathrm{~cm}^{-1}$ y a $1493 \mathrm{~cm}^{-1}$ debido a vibraciones anulares de estiramiento de las estructuras Quininoide (Q) y Bencenoide (B) respectivamente. Presenta, además, una banda de absorción a $1302 \mathrm{~cm}^{-1}$ correspondiente a la deslocalización de electrones $\pi$ inducidos en el polímero por protonación y, consecuentemente, una banda de absorción a 1247 $\mathrm{cm}^{-1}$ también correspondiente a la forma protonada. El espectro exhibe una banda de fuerte intensidad y ensanchada $1144 \mathrm{~cm}^{-1}$ que puede asignarse a la vibración de la estructura $-\mathrm{NH}^{+}$e indica la existencia de cargas positivas en la cadena.

La presencia de una banda de absorción a $878 \mathrm{~cm}^{-1}$ indica la presencia de contraiones $\mathrm{HSO}_{4}^{-}$que no han sido removidos, y la banda a $821 \mathrm{~cm}-1$ es característica de torsiones $\mathrm{C}-\mathrm{H}$. Estas características espectrales indican que la muestra obtenida en las condiciones establecidas se corresponde con polianailina protonada.

Como contrapartida, el espectro presenta una banda a $1376 \mathrm{~cm}^{-1}$, típica de una polianilina (PANI) emeraldina base, atribuida a una vibración de estiramiento $\mathrm{C}-\mathrm{N}$ en el anillo quininoide.

Por lo tanto, si bien la estructura indica la presencia de polianailina protonada, podría presentarse en equilibrio con la correspondiente esmeraldina base.

En la figura 2 se analiza en forma comparativa la muestra de PANI obtenida por método electroquímico empleando una corriente de 0,5 A y la muestra obtenida químicamente en medio ácido clorhídrico correspondiente al trabajo presentado en esta publicación (Espinoza et al. 2018). En el espectro FTIR se observan las bandas de absorción características a $1575-1488 \mathrm{~cm}^{-1}$ vibraciones anulares de estiramiento de las estructuras Quininoide (Q) y Bencenoide (B) respectivamente. La intensidad de esas bandas da una idea del estado de oxidación de la polianilina, en este caso nos indica la presencia de esmeraldina. La ausencia de la banda a 1144 $\mathrm{cm}^{-1}$ correspondiente a la vibración de la estructura $-\mathrm{NH}^{+}$(existencia de cargas positivas en la cadena), es decir, esmeraldina no dopada. La aparición de una banda de absorción a $1450 \mathrm{~cm}^{-1}$ se presenta en productos obtenidos en baja acidez.

Comparando los métodos químico y electroquímico, se puede observar que se obtiene esmeraldina con ambos métodos, pero a través del proceso de oxidación química se verifica la obtención de esmeraldina base.

Para finalizar con el análisis FTIR se observan en la Figura 3 (a, b, c, d) cuatro muestras de polianilina (PANI) obtenidas por método electroquímico utilizando intensidad de corriente de: a) $0,5 \mathrm{~A}, \mathrm{~b}$ ) $1 \mathrm{~A} \mathrm{c}$ ) $1 \mathrm{~A}$ - posteriormente suspendido en un baño de $\mathrm{HCl}$ diluido, d) $1 \mathrm{~A}$ - posteriormente suspendido en un baño de $\mathrm{NH} 3$ diluido.

Los espectros presentan las bandas características entre $1560-1588 \mathrm{~cm}^{-1}$ y $1480-1500 \mathrm{~cm}^{-1}$ por vibraciones anulares de estiramiento de las estructuras Quininoide (Q) y Bencenoide (B).

Tanto la forma ácida como la tratada en condiciones normales presentan un hombro a $1610-1615 \mathrm{~cm}^{-1}$ característicos de muestras protonadas, debido a vibraciones $\mathrm{C}=\mathrm{C}$ en la cadena polimérica. 


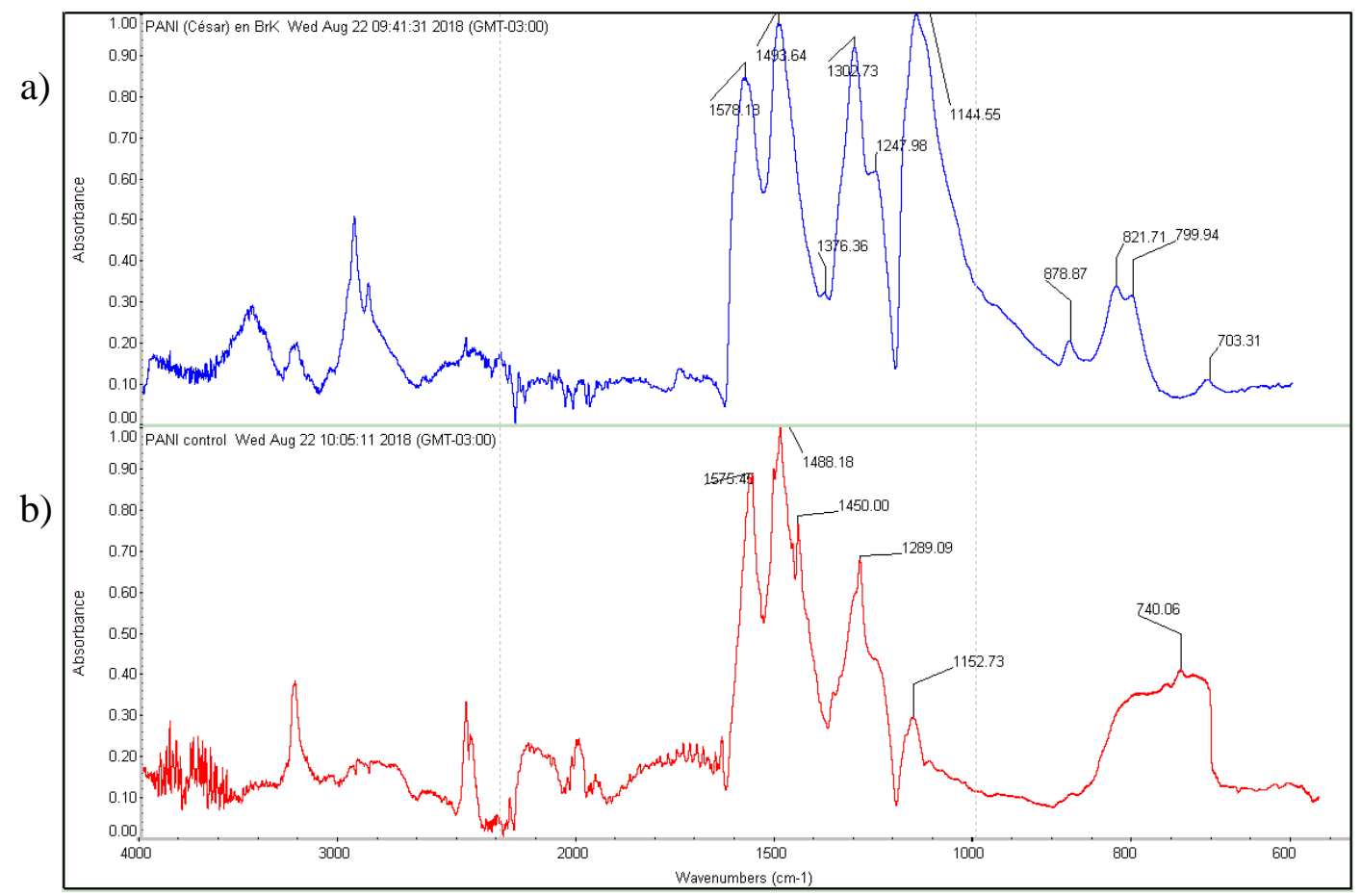

Figura 2: a) Espectros FTIR de PANI obtenida por método electroquímico empleando una corriente de 0,5 A b) PANI obtenida químicamente dopada con ácido clorhídrico

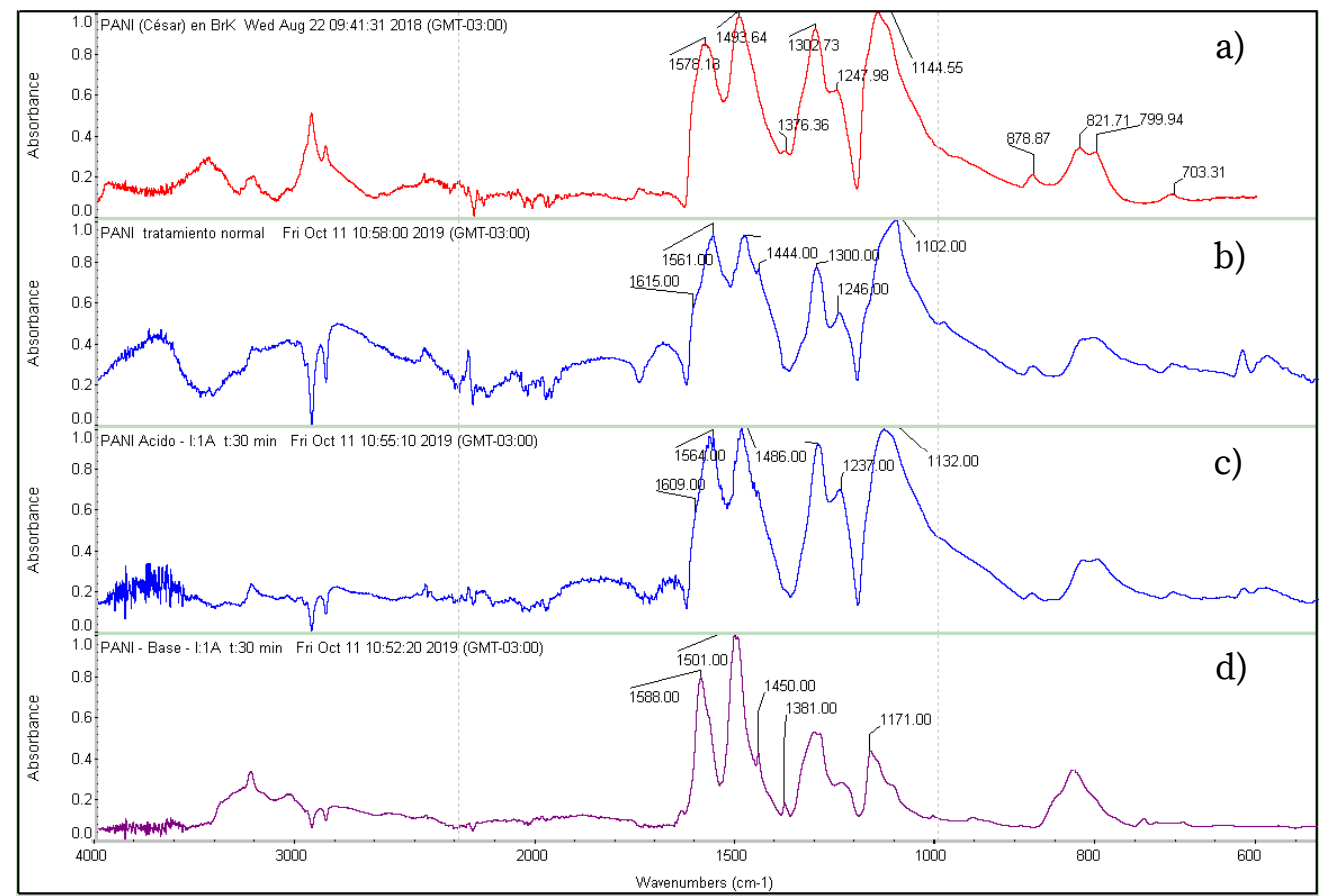

Figura 3: Espectros FTIR de polianilina (PANI)

Las muestras a y b, presentan un perfil concordante con la forma protonada de polianilina (PANI).

Se observa además la aparición de una banda de absorción a $1444 \mathrm{~cm}^{-1}$ en la muestra b, y la misma banda en el espectro de PANI almacenada en condiciones de basicidad, banda característica presente en productos obtenidos en baja acidez o en condiciones de alcalinidad. 
La presencia de una banda de absorción a $1381 \mathrm{~cm}^{-1}$, la ausencia de la banda característica de la presencia de contraión y la desaparición de la banda asignada a la vibración de la estructura $-\mathrm{NH}^{+}$indican en el espectro que la muestra resguardada en medio básico se correspondería con polianilina (PANI) emeraldina base.

Comparando los espectros de la figura 3, se puede concluir que trabajar a diferentes intensidades de corriente no afecta a la obtención de polianilina dopada.

La intensidad de la banda en muestra b y c, de debe a que aumenta el dopado de la muestra de esmeraldina, ya que la muestra se mantuvo en un baño de $\mathrm{HCl}$ diluido.

La poca intensidad en la banda a $1171 \mathrm{~cm}^{-1}$, se debe a que la muestra d se sumergió en un baño de amoniaco, obteniendo así esmeraldina base.

\section{2- Microscopía electrónica de barrido (SEM)}

Un Microscopio Electrónico de Barrido (SEM, por sus siglas en inglés) se encuentra principalmente compuesto por un emisor de haz de electrones el cual viaja a través de una columna y diferentes lentes electromagnéticas. Un sistema de deflexión permite manipular el haz de electrones para poder llevar a cabo un barrido superficial de la muestra. Una vez que los electrones incidentes interaccionan con la superficie de la muestra se generan diferentes señales: electrones secundarios, electrones retro-dispersados, rayos x, entre otras. Estas señales son capturadas por distintos tipos de detectores, ayudando a obtener información morfológica y de composición química superficial de la muestra.
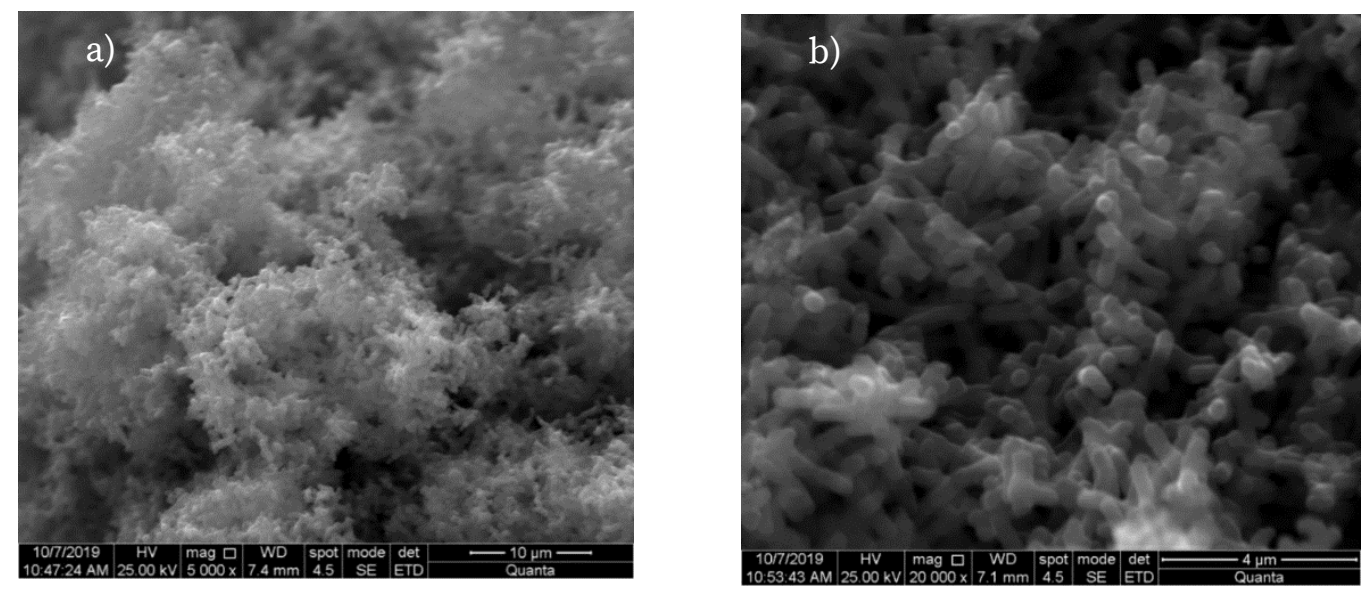

Figura 4: a) Micrografía SEM de polvos de ES 5000 X, b) Micrografía SEM de polvos de ES 20000 X

\section{Resultados}

- En el proceso electroquímico se pudo determinar que la variación de la intensidad de corriente no afecta a la obtención de PANI. Puede confirmarse esta aseveración con los espectros de la Figura 3 a) y 3 b), donde se observa las bandas características del PANI dopado.

- El residuo generado en el proceso efectuado por vía electroquímica ha resultado ser menor que el generado por vía química.

El PANI obtenido por vía electroquímica presenta partículas cilíndricas uniformes (Figura 4)

- La polimerización electroquímica es conveniente cuando se desean sintetizar películas delgadas. En cambio la polimerización química se prefiere cuando se requiere una producción a mayor escala. El espesor de las películas se puede controlar variando el tiempo y se pueden generar bicapas o multicapas sobre la superficie de un electrodo con el fin de obtener un material funcionalizado. Esta ventaja resulta ser de gran importancia para el futuro de este trabajo, ya que el objetivo siguiente consistirá en depositar PANI sobre una membrana comercial, para formar un material compuesto. 


\section{Referencias}

Heinze J. et al. (2010). Electrochemistry of Conducting Polymers - Persistent Models and New Concepts. Chemical Reviews, Vol.110, No.8, (June 2010), pp. 4724-4771 ISSN0009-2665.

Espinoza C. et al., (2018). Preparación y caracterización de un polímero conductor: polianilina (PANI), Proyecciones, Publicación UTN BA, Vol. 16, N² 2, (Octubre 2018), ISSN 1667 - 8400.

Gvozdenovic M. et al., (2011). Electrochemical Polimerization of Aniline, Electropolymerization, Dr. Ewa Schab-Balcerzak (Ed.), ISBN: 978-953-307-693-5, InTech, University Campus STeP Ri Slavka Krautzeka 83/A, 51000 Rijeka, Croatia.

Hussain A. and Kumar A., (2003). Electrochemical synthesis and characterization of chloride doped polyaniline. Bulletin of Material Science, Vol. 26, No. 3, (April 2003), pp. 329-334 ISSN0250-4707.

Wallace G. et al., (2009). Conductive Electroactive Polymers, CRC Press, Taylor \& Francis Group, ISBN 978-14200-6709-5, Boca Raton.

Inzelt G.(2008). Conducting Polymers - A New Era in Electrochemistry, Springer

Verlag, ISBN 978-3-540-75929-4, Berlin, Heidelberg.

Park S. and Joong H., (2005). Recent Advances in Electrochemical Studies of $\pi$-Conjugated Polymers. Buillten of the Korean Chemical Society, Vol. 26, No. 5, (May 2005), pp. 697-706 ISSN0253-2964.

Gupta V. \& Miura N. (2005).Large-area network of polyaniline nanowires prepared by potentiostatic deposition process. Electrochemistry Communications, Vol. 7, No. 10, (October 2005), pp. 995-999, ISSN1388-2481.

Fedorko P. et al., (2010). New analytical approach to the insulator-metal transition in conductive polyaniline. Synthetic Metals, Vol. 160, No. 15-16, (August 2010), pp. 1668-1671, ISSN0379-6779.

Genies E. et al., (1990). Polyaniline: A historical survey. Synthetic Metals, Vol. 36, No. 2, (June 1990), pp. 139182, ISSN0379-6779.

Pron A: and Rannou P., (2002). Processible conjugated polymers: from organic semiconductors to organic metals and superconductors. Progress in Polymer Science, Vol. 27, No. 1, (February 2002), pp. 135-190, ISSN0079-6700.

Tanaka et al., (1989). Molecular and electronic structures of doped polyaniline. Synthetic Metals, Vol. 29, No. 1, (March 1989), pp. 175-184, ISSN0379-6779. 